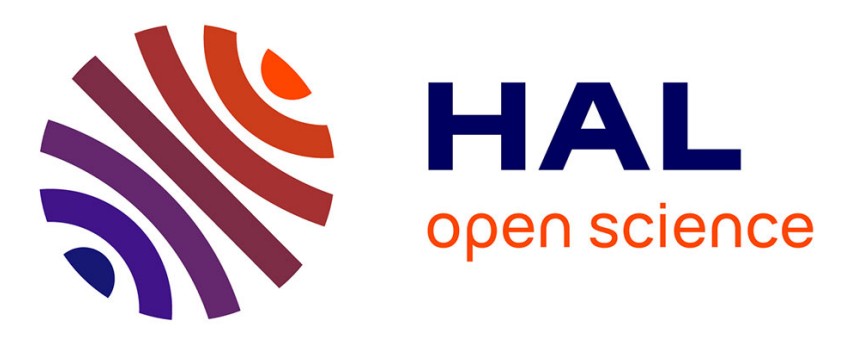

\title{
Identification of the viscoelastic properties of the tBA/PEGDMA polymer from multi-loading modes conducted over a wide frequency-temperature scale range
}

Pauline Butaud, Morvan Ouisse, Vincent Placet, Franck Renaud, Thomas Travaillot, Anne Maynadier, Gael Chevallier, Fabien Amiot, Patrick Delobelle, Emmanuel Foltete, et al.

\section{- To cite this version:}

Pauline Butaud, Morvan Ouisse, Vincent Placet, Franck Renaud, Thomas Travaillot, et al.. Identification of the viscoelastic properties of the tBA/PEGDMA polymer from multi-loading modes conducted over a wide frequency-temperature scale range. Polymer Testing, 2018, 69, pp.250 - 258. 10.1016/j.polymertesting.2018.05.030 . hal-02130068

\section{HAL Id: hal-02130068 https://hal.science/hal-02130068}

Submitted on 15 May 2019

HAL is a multi-disciplinary open access archive for the deposit and dissemination of scientific research documents, whether they are published or not. The documents may come from teaching and research institutions in France or abroad, or from public or private research centers.
L'archive ouverte pluridisciplinaire HAL, est destinée au dépôt et à la diffusion de documents scientifiques de niveau recherche, publiés ou non, émanant des établissements d'enseignement et de recherche français ou étrangers, des laboratoires publics ou privés. 


\author{
Material Properties \\ Identification of the viscoelastic properties of \\ the tBA/PEGDMA polymer from multi-loading modes \\ conducted over a wide frequency-temperature scale \\ range \\ P. Butaud a, M. Ouissea, V. Placet ${ }^{a}$, F. Renaud ${ }^{b}$, T. Travaillota, A. Maynadiera, \\ G. Chevalliera, F. Amiota ${ }^{a}$ P. Delobellea, E. Foltête ${ }^{a}$, and C. Rogueda-Berriet ${ }^{a}$ \\ aUniv. Bourgogne Franche-Comté FEMTO-ST, CNRS/UFC/ENSMM/UTBM, Department of Applied \\ Mechanics 24, Chemin de l'Epitaphe 25000 Besançon - FRANCE \\ ${ }^{b}$ Supmeca LISMMA, 3 rue Fernand Hainaut - 93407 Saint Ouen Cedex - FRANCE
}

\begin{abstract}
The viscoelastic properties of tBA/PEGDMA, which is a Shape Memory Polymer (SMP), were characterized over wide bands of frequency and temperature. The continuity of the viscoelastic properties identified according to the frequency, the temperature, the loading mode and the test scale was assessed. Seven experimental methods were used, from quasi-static to high frequencies, in tensile, shear and compression, from the nanoscopic scale to the macroscopic scale. The storage modulus and the loss factor of the SMP were evaluated and compared from one method to the other. The comparison between the various tests was done based on measurements obtained with a Dynamic Mechanical Analysis and extrapolated through the TimeTemperature Superposition principle. All the data gathers on a unique master curve. For the various experimental methods, it appears that all the viscoelastic properties are consistent even if different scales and loading modes are involved. This wide band characterization makes it possible to determine the mechanical viscoelastic properties of the tBA/PEGDMA in order to use it in structural applications. The experimental methods used in this paper combine commercial methods and in-house high-tech methods. It should be emphasized that the set of experiments covers effective measurements from $10^{-4}$ to $10^{6} \mathrm{~Hz}, 0$ to $90 \mathrm{C}$, strain levels from $10^{-4 \%}$ to $5 \%$, at nano, micro and macro scales on the same material.
\end{abstract}


Keywords: shape memory polymer, viscoelastic, characterization, wide-band, dynamic, experimental methods

Email address: pauline.butaudeuniv-fcomte.fr (P. Butaud)

\section{Introduction}

Structural lightening is a topic of concern for many industrial sectors. In particular, many metal structures have recently been replaced by composite elements in the fields of aeronautics, aerospace, land and water transport. The use of composite materials has been increasingly considered as they often exhibit higher specific stiffness and lower density compared to conventional materials. However, there are various scientific challenges associated with these new materials, including manufacture, aging and resistance to complex environments. Additionally, they typically exhibit poor dynamic behaviour because of their high-rigidity and low-damping properties. The control and reduction of noise and vibration are highly important issues. Passive damping treatments that constitute efficient, lowcost and robust solutions have been extensively investigated, and extensive research work has focused on composite structures with embedded viscoelastic materials to damp vibrations [1-4]. For this purpose, precise mechanical characterisation of the viscoelastic properties of polymers is needed.

Typically, to obtain the viscoelastic mechanical properties of a polymer, dynamic mechanical analysis (DMA) is conducted on a small band of frequencies and for several temperatures [5-7]. Based on the Williams-Landel-Ferry (WLF) [8] or the Arrhenius laws [9], and in accordance to the time-temperature superposition (TTS) model, DMA measurements are conducted to obtain the mechanical behaviour of the polymer for large bands of frequency 
and temperature. The extrapolated properties that are obtained can then be used to design composite structures. However, some limits of this type of characterisation have been reported in several works. For example, Hutcheson et al. [10] reported errors up to one order of magnitude in the determination of the viscoelastic properties of materials owing to the effects of instrument compliance. Diani et al. [11] also warned against dynamic torsion tests where the storage modulus and damping factor can be overestimated depending on the dimensions of the chosen specimen. Limits have also been published by Butaud et al. [12] who reported that the extrapolation of the mechanical properties on a wider band of temperatures and/or frequencies is highly dependent on the chosen TTS model. Moreover, these viscoelastic properties are often obtained only for a certain loading mode. However, the polymer is ultimately integrated into a complex structure, which is exposed to multiple loading cycles in a complex environment in terms of frequency and temperature. Therefore, this study investigates the validity of the TTS principle applied to different loading modes and scales, and the complementary nature of several experimental methods.

This investigation is focused on the viscoelastic characterisation of tBA/PEGDMA, which is the polymer material used by Butaud et al. [4] as a sandwich core to tune the damping of a composite structure. It is a shape memory polymer (SMP), and thus presents mechanical properties that are very sensitive to temperature and frequency. Indeed, efficient shape memory effects are associated with fast transitions between stable glassy and rubbery states with a large elasticity gap, inducing high-loss factor values at glass transition $[13,14]$. This type of material seems to be a good candidate for testing different experimental characterisation methods.

Mechanical characterisations of tBA/PEGDMA are already available in the literature. Nair et al. reported a shape memory effect analysis [15], Yakacki et al. [16] conducted a study on the shape recovery of SMP, Wornyo et al. executed nano-indentation tests [17], while Ortega et al. performed dynamic mechanical analysis at $1 \mathrm{~Hz}$ [18]. Nevertheless, all these mechanical properties have not obtained for the same wt $\%$ of the tested components, and hence the results are not really comparable. This study focuses on 
the experimental investigation of tBA/PEGDMA at $95 / 5 \mathrm{wt} \%$ over a wide band of frequencies and temperatures using various experimental methods, at different scales and with different loading modes. Similar to the work of Zeltmann et al. [19], where good agreement was found between the prediction of the TTS model and DMA and quasi-static tests at various strain rates, we propose herein to extend this questioning on the equivalence of various experimental methods. Low frequencies were investigated using a quasi-static tensile test. The medium frequencies were tested using modal analyses. The high frequencies were achieved by DMA on a visco-analyser high-frequency device. Other methods, such as nano-indentation, ultrasonic testing and scanning microdeformation microscopy (SMM) were also used to achieve smaller loading scales. Where possible, these tests were carried out at different temperatures in order to scan a wider range of mechanical properties.

The remainder of the work is organised as follows. The Material and methods section lists methodological details on material preparation and the experimental setups of all the characterisation methods. The Results section details the results obtained using the different methods. Finally, the Discussion section considers the complementary nature of these various experimental methods.

\section{Material and methods}

\subsection{Material and specimen preparations}

The polymer tBA/PEGDMA that was originally studied by Srivastava et al. [20] was evaluated in this study. Work was executed at the FEMTO-ST Institute in the Department of Applied Mechanics in accordance with the procedure originally described in Yakacki et al. [16]. The chemicals were provided by SigmaAldrich and used as received without any purification. The shape memory polymer was synthesised by manually mixing $95 \mathrm{wt} \%$ of the monomer tert-Butyl Acrylate (tBA), with $5 \mathrm{wt} \%$ of the crosslinking agent poly(ethylene glycol) dimethacrylate (PEGDMA) (with a typical molecular weight $M n=550 \mathrm{~g} / \mathrm{mol}$ ). The photoinitiator, 2,2-dimethoxy-2-phenylacetophenone (DMPA), was added to the solution at a concentration of $0.5 \mathrm{wt} \%$ of the total weight. The liquid mixture was then injected between two glass 
slides. A plastic seal was used as a spacer to ensure a given thickness. The polymerisation was initiated by exposing the solution to UV light for $10 \mathrm{~min}$ and was completed by heating the polymer at $90^{\circ} \mathrm{C}$ for $1 \mathrm{~h}$. The plates obtained were machined as required for the different mechanical tests.

tBA/PEGDMA is a thermoset amorphous polymer. A DSC analysis highlighted the absence of crystallites and full cross-linking of the polymer. Moreover, optical imaging analyses have not shown any polymer heterogeneity. Finally, it should be emphasised that tBA/PEGDMA is thermo-rheologically simple, as mentioned in Butaud et al. [21].

\subsection{Dynamic Mechanical Analysis}

Samples for DMA tests were cut to the dimensions of $29 \times 6 \times 3$ $\mathrm{mm}^{3}$. Viscoelastic properties (storage modulus $E^{\prime}$ and loss factor $\tan (\delta)$ ) were measured using a METRAVIB DMA50 apparatus at temperatures that varied in $5^{\circ} \mathrm{C}$ steps (near the glass transition temperature), or at $10^{\circ} \mathrm{C}$ steps (far from the glass transition) at a heating rate of $2^{\circ} \mathrm{C} / \mathrm{min}$, between $0^{\circ} \mathrm{C}$ and $90^{\circ} \mathrm{C}$. The frequency of the excitation varied from $0.1 \mathrm{~Hz}$ to $180 \mathrm{~Hz}$. A sinusoidal tensile displacement was applied on the sample with a peak-to-peak amplitude of $10 \mu \mathrm{m}$, thus ensuring linear viscoelastic behaviour.

The thermal field was monitored by infrared thermography using a CEdip Jade III MWIR camera to visualise the possible overheating of the sample. The measurements were performed during the DMA test but only between 0.1 and $10 \mathrm{~Hz}$ at ambient temperature. The temperature variations observed, measured noise and Narcissus effect were not significant, and no temperature evolution could be related to mechanical loading.

\subsection{Quasi-static tests}

Dumbbell shaped specimens were produced for quasi-static tensile tests, the gauge length was $33 \mathrm{~mm}$ and the rectangular cross-section was $3 \times 6 \mathrm{~mm}^{2}$. The quasi-static tensile tests were carried out in accordance with ASTM D 638-03 [22] using a commercial testing machine (Instron 6025) with a $\pm 5 \mathrm{kN}$ capacity load cell . The specimens were clamped using wedge action grips and were subjected to displacement control feedback at $1 \mathrm{~mm} / \mathrm{min}$ and $5 \mathrm{~mm} / \mathrm{min}$, i.e. at respective strain rates of $0.05 \% \mathrm{~s}^{-1}$ and 
$0.25 \% \mathrm{~s}^{-1}$. The tests were performed at ambient temperature $\left(21^{\circ} \mathrm{C}\right.$ $\pm 1^{\circ} \mathrm{C}$ ). The engineering longitudinal strain was measured with a laser extensometer (EIR LE05) with a displacement measurement accuracy of $5 \mu \mathrm{m}$. An optical camera (Ueye, 1 Mpixel, 8 bits) was used to assess the displacement fields in the gauge part of the specimen using digital image correlation (DIC) with image acquisition rate of $1 \mathrm{~Hz}$. DIC was performed with a home-made global correlation cod, implemented in Octave, as previously described by Rethoré et al. [23].

\subsection{Modal analysis}

Modal analysis was completed with dynamic mechanical experimental tests with broadband random excitation, thus allowing the identification of the modes of a structure. Additionally, a finite element model (FEM) of the structure was established and a model updating procedure [24] provided the material mechanical properties at the modal frequencies of the structure. This method estimated the storage modulus and the loss factor of the tBA/PEGDMA over a wide frequency band and for several temperatures.

A plate with dimensions $157.7 \times 45.5 \times 3.1 \mathrm{~mm}^{3}( \pm 0.1 \mathrm{~mm})$ was used. The tBA/PEGDMA rectangular plate was suspended with soft springs to realise free-free conditions. The external force was applied using a voice-coil actuator with a permanent magnet glued at one of the corners of the plate. The set was placed in a thermal chamber with a temperature control at $\pm 2^{\circ} \mathrm{C}$ and with temperatures that ranged from $22^{\circ} \mathrm{C}$ to $43^{\circ} \mathrm{C}$ (in $7^{\circ} \mathrm{C}$ increments). A broadband random excitation was applied between 150 and 3000 $\mathrm{Hz}$. The plate response was measured with a laser vibrometer and successively focused on 28 reflecting stickers spread on the plate. The modal frequencies, modal damping coefficients and mode shapes were then identified using Modan ${ }^{\odot}$ (a homemade modal analysis software) from the measured frequency response functions (FRFs).

A finite element model of the plate was established on Patran ${ }^{\mathrm{TM}} 2012$ under the hypothesis that the material was homogeneous and isotropic, with a Poisson's ratio $v=0.37$ (determined from quasi-static tests), a mass density $\rho=1000$ 
$\mathrm{kg} / \mathrm{m}^{3}$ (determined by a pycnometer), and a predefined Young's modulus. The magnet was included in the numerical model $(E=$ $210000 \mathrm{MPa}, v=0.33, \rho=7460 \mathrm{~kg} / \mathrm{m}^{3}$ ). Finally, a model-test correlation was performed on AESOPc software and the model updating process allowed the storage modulus of the tBA/PEGDMA at the modal frequencies. The loss factor was directly deduced from the modal damping $\xi$ without the use of the finite element model with

$$
\tan (\delta)=2 \xi .
$$

This procedure was detailed and discussed in Butaud et al. [12].

\subsection{High Frequency Viscoanalysis}

The high-frequency visco-analyser (HFV) developed by F. Renaud et al. [25] was designed to measure the mechanical properties of visco-elastic materials over a wide frequency range, that is, from $[100-200 \mathrm{~Hz}]$ to $[2000-5000 \mathrm{~Hz}]$ according to the rigidity of the material. Figure 1 shows a picture of the HFV and its schematic diagram. Four samples are clamped between the jaws and the sample holders. This assembly was symmetrical and made it possible to subject samples to pure shear without bending. A normal static preload was applied through an M6 bolt in the centre of the jaws. The shearing of the samples was obtained by moving the holders in phase opposition. To achieve this, two actuation chains that consisted of a piezoelectric actuator and a force sensor were placed between the holders. The piezoelectric actuators (PI842.10) can reach frequencies as high as $10 \mathrm{kHz}$. However, the upper frequency limit used for characterisation was determined by the first natural resonance frequency of the HFV. The HFV was suspended by elastic bands to realise free-free conditions. Dynamic forces were measured using force sensors (B\&K 8200). The pre-load force was measured by a load-cell located under the lower jaw. Six piezoelectric accelerometers were placed on the jaws and the holders in order to measure the accelerations of the four samples.

Samples for the HFV tests were cut to the dimensions of $20 \times 30$ $\times 2.5 \mathrm{~mm}^{3}$. The experimental tests on the tBA/PEGDMA were carried out in a thermal chamber. The force sensors limited the 
maximum temperature to $80^{\circ} \mathrm{C}$, and the temperature was thus varied from $20^{\circ} \mathrm{C}$ to $80^{\circ} \mathrm{C}$ (in $10^{\circ} \mathrm{C}$ increments). It was observed after the first temperature cycle that the samples stick to the aluminium, so the pre-load bolt was removed for conduct of the experimental tests.

The post-processing of the measurements, detailed in [25], yielded the complex shear modulus $G^{*}$ according to the temperature and the frequency (that ranged between 200 and $2000 \mathrm{~Hz}$ ). In order to compare these measurements with the other experimental tests, the complex modulus $E^{*}$ was estimated by

$$
E^{*}(\omega)=2 G^{*}(\omega)(1+v),
$$

where $\omega=2 \pi f$ is the excitation pulsation, and $v=0.37$ is the Poisson's ratio determined by quasi-static tests. The Poisson's ratio was supposed to be constant as a function of frequency and temperature. This hypothesis is discussed in the Results section.

It should be emphasised that the experimental apparatus used in this section was under development, and the objective here was twofold: a) acquisition of new tBA/PEGDMA data, and b) determination of the limits of the experimental method. The HFV bench test was initially designed for silicone materials which have a storage modulus of approximately $100 \mathrm{MPa}$ versus corresponding values of $2500 \mathrm{MPa}$ for tBA/PEGDMA. The accelerometer data were measured during the bench test and not directly on the sample. It is thus difficult to dissociate the stiffness of the system and the stiffness of the sample through the analytic model. In our case, the estimated modulus for low temperatures may be underestimated. Nevertheless, the HFV can provide reliable measurements at high temperatures.

\subsection{Nano-indentation tests}

Nano-indentation involves the penetration of the material with an indenter whose tip radius is nano-metric, followed by its retraction. The analysis of the contact stiffness during unloading provides access to the indentation modulus of the material. To obtain its elastic properties, two methods were used: 
- the classical, continuous, multi-cycle (CMC), charge-discharge method proposed by W. C. Oliver et al. [27] which provides a static measurement

- the continuous stiffness method (CSM) technique described by X. Li et al. [28], which adds a harmonic force to the classical method and provides a frequency-dependent measurement using harmonic loading

These two methods yield a reduced elastic modulus $E_{r}$ in accordance with

$$
E_{r}=\frac{E}{1-v^{2}} .
$$

In order to estimate the elastic modulus of the tBA/PEGDMA, the quasi-static Poisson's ratio value was used $(v=0.37)$.

Nano-indentation tests were carried out using an Anton-Paar nanoindenter equipped with a conical tip at a loading speed of $4.2 \mu \mathrm{N} / \mathrm{s}$. The study was conducted following the CMC after $300 \mathrm{~s}$ at the maximum load $(\operatorname{Pmax}=500 \mu \mathrm{N})$ to inhibit creep deformation. The frequency of the quasi-static test was estimated to be $0.01 \mathrm{~Hz}$ based on

$$
f=\frac{1}{h_{\max }} \frac{d h}{d t}=10^{-2} s^{-1},
$$

where the maximum indentation depth $h_{\max }=350 \mathrm{~nm}$, and the maximum indentation speed $\frac{d h}{d t}=3.5 \mathrm{~nm} / \mathrm{s}$. In accordance with the CSM technique, the sample was tested with sinusoidal loading at 2, 20,80 , and $200 \mathrm{~Hz}$. The tests were conducted at ambient temperature $\left(20^{\circ} \mathrm{C} \pm 1^{\circ} \mathrm{C}\right)$.

\subsection{Scanning Microdeformation Microscopy (SMM)}

Some experiments at high frequencies were performed using SMM, which is a type of a force contact microscope [29]. The SMM tests used a vibrating tip acoustic microscope developed at the FEMTO-ST Institute [30]. The SMM was based on a vibrating sensor (resonator) consisted of a support, a bi-morph piezoelectric transducer, a micro-lever and a sapphire tip with a radius of $15 \mu \mathrm{m}$ (Figure 2). The principle of the SMM was to bring the tip into contact with the tBA/PEGDMA sample, and to excite the resonator by frequency sweeping to measure the micro-lever bending modes. 
The resonant frequencies depend on the geometry of the vibrating sensor and on the boundary conditions, and thus on the mechanical properties of the sample material that is in contact with the tip. According to the stiffness of the material, the resonant frequencies were modified. A dynamic model, introduced by Le Rouzic et al. [31], allows estimation of the mechanical properties (storage modulus and Poisson's ratio) for the sample. The acoustic microscopy test was performed at room temperature $\left(20^{\circ} \mathrm{C} \pm 1^{\circ} \mathrm{C}\right)$ on a $3 \mathrm{~mm}$ thick sample. The support height $\mathrm{z}$ (Figure 2) was set to $0.27 \mu \mathrm{m}$. The resonance frequencies measured 'in contact' with the tBA/PEGDMA were $17,850 \mathrm{~Hz}, 41,500 \mathrm{~Hz}, 49,600 \mathrm{~Hz}$, and 106,400 $\mathrm{Hz}$.

\subsection{Ultrasonic waves}

The characterisation of the tBA/PEGDMA polymer by ultrasonic waves was conducted at room temperature $\left(20^{\circ} \mathrm{C} \pm 1^{\circ} \mathrm{C}\right)$. Transceiver transducers of longitudinal waves (Olympus V110-RM at $5 \mathrm{MHz}$ ) and transverse waves (Olympus V154-RM at $2.25 \mathrm{MHz}$ ) were applied on a $3 \mathrm{~mm}( \pm 0.1 \mathrm{~mm})$ plate with gel-type coupling to ensure good diffusion of the waves in the material. The measurements of the longitudinal and transverse wave propagation velocities were estimated in accordance to the hypothesis of a purely elastic behaviour, and allowed estimation of the Lamé coefficients and the Young's modulus and Poisson's ratio of the tBA/PEGDMA.

\section{Results}

\subsection{DMA and visco-elastic modelling}

The DMA measurements are shown in Figure 3. As shown, the tBA/PEGDMA properties vary with temperature and frequency. The storage modulus $E$ changed by three orders of magnitude. The glass transition temperature varied from 45 to $65^{\circ} \mathrm{C}$ depending on the frequency. The loss factor $\tan (\delta)$ reached values as high as 2.5 .

The master curve generated based on the DMA measurements (Figure 4) was obtained based on the use of a least-squares curve fitting technique. The temperature evolution of the shift factor $a_{T}$ was expressed according to the Williams-Landel-Ferry (WLF) law [8] in accordance with 


$$
\log \left(a_{T}\right)=\frac{-C_{1}^{0}\left(T-T_{0}\right)}{-C_{2}^{0}+\left(T-T_{0}\right)}
$$

with $C_{1}^{0}=28.28^{\circ} \mathrm{C}$ and $C_{2}^{0}=12.52^{\circ} \mathrm{C}$ at a reference temperature $T_{0}=20^{\circ} \mathrm{C}$.

In order to compare the different experimental methods, a model that describes the rheological properties of a viscoelastic material was used to extrapolate the data. The 2S2P1D model, the name of which comes from the abbreviation of the combination of two springs, two parabolic creep elements and one dashpot [32], allows estimation of the complex modulus based on

$$
E^{*}(i \omega \tau)=E_{0}+\frac{E_{\infty}-E_{0}}{1+\gamma(i \omega \tau)^{-k}+(i \omega \tau)^{-h}+(i \omega \beta \tau)^{-1}},
$$

where $k$ and $h$ are exponents with $0<k<h<1, \gamma$ and $\beta$ are constants, $E_{0}$ is the rubber modulus when $\omega \rightarrow 0$, and $E_{\infty}$ is the glassy modulus when $\omega \rightarrow \infty$. Equivalently, $\tau$ is the characteristic time, estimated based on the TTS principle as $\tau(T)=a_{T}(T) \cdot \tau_{0}$. The identified parameters are given in Table 1.

\begin{tabular}{ccccccc}
\hline$E_{0}(\mathrm{MPa})$ & $E_{\infty}(\mathrm{MPa})$ & $k$ & $h$ & $\gamma$ & $B$ & $\tau_{0}(\mathrm{~s})$ \\
\hline 1.01 & 2190 & 0.17 & 0.79 & 1.43 & $3.1 \mathrm{e}+4$ & $2.13 \mathrm{e} 17$ \\
\hline
\end{tabular}

Table 1: 2S2P1D model parameters for the tested tBA/PEGDMA polymer.

Figure 4 shows the master curve of the storage modulus and the loss factor of the tBA/PEGDMA compared to the 2S2P1D model. The properties of the $\mathrm{tBA} / \mathrm{PEGDMA}$ are plotted for the reference temperature of $20^{\circ} \mathrm{C}$ because the other experimental methods are often conducted at ambient temperature. More details about the dynamic mechanical analysis of the tBA/PEGDMA specimen and the development of the modelling of the visco-elastic behaviour can be found in $[21,4]$. The following sections present the results of the various experimental methods.

\subsection{Quasi-static results}

The apparent Young's modulus was determined based on quasistatic tests. It is qualified as an 'apparent' index because of the dependency of the modulus on frequency and temperature in regard to the visco-elastic materials. 
In accordance with a one-dimensional restriction, the nominal stress $\sigma$ is defined by

$$
\sigma=F / S_{0}
$$

where $F$ is the tensile force applied to the specimen, and $S_{0}$ is the initial cross-sectional area of the specimen. The nominal strain $\varepsilon L$ is defined by

$$
\varepsilon_{L}=\Delta L / L_{0},
$$

with $L_{0}$ the original gauge length and $\Delta L$ the elongation of $L_{0}$ during the test.

Figure 5(a) shows the evolution of the nominal stress $\sigma$ as a function of the strain $\varepsilon_{L}$ for the two loading rates. The sample tested at $5 \mathrm{~mm} / \mathrm{min}$ exhibits brittle failure behaviour with a fracture limit of approximately $36 \mathrm{MPa}$ and a fracture strain of 3.4 $\%$. The sample tested at $1 \mathrm{~mm} / \mathrm{min}$ exhibits ductile failure behaviour, where an elastic domain is observed for stress values up to $32 \mathrm{MPa}$ and for $3 \%$ of strain, followed by flow with the appearance of localised necking in regions where the sample deforms with almost constant force until fracture at an approximate strain of $65 \%$. The fracture behaviour of the tBA/PEGDMA seems to be strongly dependent on the loading rate.

Therefore, the apparent Young's modulus is found to be independent of the tensile speed for the two tested loading rates (Figure 5(b)). An initial tangent modulus $E_{0}=2180 \mathrm{MPa}$ was measured based on the slope of the linear part of the $\sigma-\varepsilon_{L}$ curve for $\varepsilon_{L}=[0-0.5 \%]$. The modulus can also be determined by using the slope of the tangent to the $\sigma-\varepsilon_{L}$ curve at a defined strain rate. Thus, depending on the method chosen for the estimation, and depending on the selected strain range, the apparent Young's modulus can vary from $1850 \mathrm{MPa}$ to $2500 \mathrm{MPa}$, i.e. by $\pm 15 \%$ around $E_{0}$.

Similar to many other polymers, the determination of the Young's modulus for tBA/PEGDMA is not trivial, and even the notion of Young's modulus does not necessarily have any meaning because the material does not exhibit perfectly linear elastic behaviour. Hereafter, the value of the initial tangent modulus $E_{0}$ will be used to define the apparent quasi-static Young's modulus of the 
tBA/PEGDMA polymer. To our knowledge, no results related to the quasi-static analysis of the tBA/PEGDMA are available in the literature, but this modulus value is consistent with the glassy storage modulus estimated by DMA.

The Poisson's ratio $v$ of the tBA/PEGDMA was determined by DIC during quasi-static tests at room temperature. According to the zone selected in the image for the DIC, and according to the tensile test studied, the Poisson's ratio was estimated at $0.37( \pm 2 \%)$. Moreover, the tests carried out have shown that, in the case of the apparent Young's modulus, the loading rate does not seem to influence the value of the Poisson's ratio. However, several studies $[33,34,35,36]$ reported the variation in the Poisson's ratio for polymers between the glassy and the rubbery states. The experimental measurement of this type of dependency can be challenging [34, 37], and has not been implemented in this study. Thus, in the following sections, it is assumed that the Poisson's ratio does not vary with temperature or frequency, and $v=0.37$ is considered when necessary for multiple frequency-temperature measurements. The quasi-static results are compared to the 2S2P1D model in the Discussion section.

\subsection{Modal analyses results}

The storage moduli $E^{\prime}$ and loss factors $\tan (\delta)$ identified through modal analyses are presented in Figure 6. Depending on the temperature of the test, 6 to 9 modes were measured between 170 to $[1000-3000 \mathrm{~Hz}]$.

The modal tests show a classical evolution of the storage modulus and loss factor with temperature, where a decrease in $E$ and an increase in $\tan (\delta)$ are found for increasing temperatures below the glass temperature. The evolution of the loss factor with frequency also seems consistent. Conversely, the behaviour of the storage modulus with respect to the frequency is unexpected. The first modes of the storage modulus seem to decrease slightly with frequency increases, and then increase again. This trend is notable for all the temperatures studied. It should be emphasised that, in addition to the uncertainties associated with the experimental procedure, the identification method can also introduce some bias in the results. Several possibilities are proposed to explain this 
unexpected trend. First, the Poisson's ratio, the value of which is necessary for the updating of the model is supposed to be frequency independent, and its value is being specified by the quasi-static tests, thus introducing some bias. Secondly, there is an expected impact owing to the non-constant thickness of the plate, which varies from $2.98 \mathrm{~mm}$ to $3.14 \mathrm{~mm}$ as a result of the manufacturing process. The influence of the boundary conditions, the updating method, the heterogeneity of density or elastic modulus, or the self-heating of the material during the test can then explain these results. By combining the various possible sources of discrepancies (geometric, thermal, and mechanical), the stability of the storage modulus could finally be determined over the frequency range of interest. The curve of Figure 6 that corresponds to $43^{\circ} \mathrm{C}$ shows the error bars associated with each mode taking into account the uncertainties of the thickness and the Poisson's ratio.

In contrast, these modal tests cannot be used to evaluate the behaviour of tBA/PEGDMA in the glass transition state because of the softening of the polymer. In order to measure the rubbery properties based on modal analyses, the Oberst's experimental test could be used [38], as defined in ASTM-1998 [39]. It is comprised of testing the visco-elastic material associated with one or two layers of rigid material, typically in a sandwiched form. The structure to be tested is then quite rigid in the rubbery phase for the conduct of the modal test. Nevertheless, this method adds uncertainties to the finite element model which become dependent, not only on the boundary conditions, but also on the mechanical properties of the added materials.

Finally, modal tests at $22^{\circ} \mathrm{C}$ yielded an estimate of the average storage modulus of $2330 \mathrm{MPa}( \pm 3.5 \%)$ over the frequency range $[170-3000 \mathrm{~Hz}]$, which was slightly higher than the value corresponding to the asymptotic behaviour of the 2S2P1D model (2190 MPa). The comparison between the modal analysis and the 2S2P1D model at each temperature for the storage modulus and the loss factor, which are in good agreement, is detailed in the Discussion section. 


\subsection{High-frequency visco-analysis results}

The storage modulus $E$ and the loss factor $\tan (\delta)$ estimated based on HFV according for several temperatures and as a function of frequency, are presented in Figure 7. The estimated storage modulus varies between $2.8 \mathrm{MPa}$ and $1540 \mathrm{MPa}$, and the loss factor between 0 and 2.1 for the temperatures spanning the range of [20$\left.80^{\circ} \mathrm{C}\right]$ and the frequency range of $[200-2000 \mathrm{~Hz}]$.

Uncertainties could arise from the preload because the mechanical properties of some polymeric materials vary with the preload [40]. This aspect remains to be explored in the case of tBA/PEGDMA. Once again, the Poisson's ratio is used in data postprocessing, and the question of its dependency on temperature and frequency still holds. A comparison of dynamic values measured in tensile and in shear tests on the same frequency band could answer this question. Unfortunately, the frequency bands do not overlap in the DMA and HFV tests, and the dependency on the Poisson's ratio to the frequency could not be observed.

Despite these uncertainties, the HFV results are in good agreement with the results obtained from the 2S2P1D model, and the general trend remains correct. The HFV is able to measure the amazing loss factor of the tBA/PEGDMA once again over $200 \%$ at the glass transition. More details on the continuity between the properties obtained with the HFV and those identified with the others experimental methods are listed in the Discussion section.

\subsection{Results from other experimental methods}

Tests on the tBA/PEGDMA polymer at ambient temperature were also carried out using nano-indentation, scanning microdeformation microscopy SMM, and ultrasonic analysis.

Nano-indentation. Storage modulus values obtained as a function of frequency are shown in Figure 8. The nano-indenter did not detect a phase shift between the excitation and the response, and therefore estimated a loss factor of zero. The storage modulus measured by nano-indentation is approximately equal to $2250 \mathrm{MPa}$ $( \pm 11 \%)$. There is a gap between the static and the dynamic measurements. Nevertheless, no conclusion can be deduced on the mechanical properties of tBA/PEGDMA based on this gap, but the 
type of method used (CMC to CSM) may explain the difference in the modulus values. Moreover, the increase in the storage modulus between $80 \mathrm{~Hz}$ and $200 \mathrm{~Hz}$ is potentially due to the resonance of the nano-indentation machine which occurs at approximately 80 Hz.

SMM. According to the SMM measurements, at the operating frequencies, the storage modulus does not depend on the excitation frequency. The storage modulus is estimated at 2630 MPa within the frequency band of [17850-106400 Hz]. This method also yields an approximate Poisson's ratio value of 0.34 .

Ultrasound. The ultrasonic tests conducted at a very high frequency estimated a Young modulus of $2200 \mathrm{MPa}( \pm 8 \%)$ and a Poisson's ratio of $0.37( \pm 2 \%)$. The transceiver's transducers of the longitudinal waves and the transverse waves did not have the same operating frequency (5 $\mathrm{MHz}$ and $2.25 \mathrm{MHz}$, respectively). However, at these frequency levels, and at room temperature, the mechanical properties of the tBA/PEGDMA were stabilised, and the frequency difference between the two transducers did not constitute a considerable hindrance.

\section{Discussion}

The results of the seven experimental methods conducted herein are plotted in Figure 9. In this figure, the storage modulus $E$ and the loss factor $\tan (\delta)$ are plotted according to the reduced frequency $f . a_{T}$ at $20^{\circ} \mathrm{C}$ using the WLF law to perform horizontal translations of the isotherm curves for each experimental test.

In order to discuss the consistency of the experimental methods, Figure 9 and Table 2, respectively, depict and list the experimental setups and results. The 2S2P1D model is consistent with the seven experimental methods. It should be emphasised that the set of experiments covers effective measurements in the range of $10^{-4}$ to $10^{6} \mathrm{~Hz}, 0$ to $90^{\circ} \mathrm{C}$, and strain levels from $10^{-4} \%$ to $5 \%$ at the nano-, micro- and macro-scales on the same material. 
In Figure 9, the blue crosses correspond to the master curve obtained by DMA and in accordance to the TTS principle with a reference temperature of $20^{\circ} \mathrm{C}$.

The dotted line corresponds to the 2S2P1D model determined from the DMA master curve.

The blue triangles correspond to the quasi-static tests. The strain rates of the specimens are chosen as a reference to define the frequency of the quasi-static tests. A modulus of $2180 \mathrm{MPa}$ is thus measured at $2.5 \times 10^{-3} \mathrm{~Hz}$ and $5 \times 10^{-4} \mathrm{~Hz}$ for a temperature of $20^{\circ} \mathrm{C}$. It is then possible to add on the DMA master curve and the associated model 2S2P1D results the measured points obtained in accordance to the quasi-static tests, as shown in Figure 9. The quasi-static tests validated the asymptotic behaviour of the 2S2P1D model at reduced frequencies. In more general terms, the quasistatic tests made it possible to quickly validate measurements using a simple test, justifying the coherent behaviour of the material.

Table 2: Experimental tests summary.

\begin{tabular}{|c|c|c|c|c|c|}
\hline Method & $\begin{array}{l}\text { Loading } \\
\text { mode }\end{array}$ & $\begin{array}{c}\text { Frequency } \\
\text { band } \\
{[\mathrm{Hz}]}\end{array}$ & $\begin{array}{c}\text { Temperature } \\
{[\mathrm{C}]}\end{array}$ & Scale & $\begin{array}{c}\text { Order of } \\
\text { magnitude } \\
\text { of strain } \\
{[\%]}\end{array}$ \\
\hline DMA & Tensile & $0.1-180$ & $0-90$ & Macroscopic & 0.1 \\
\hline Quasi-static & Tensile & $10-4-10-3$ & 20 & Macroscopic & 0.5 \\
\hline Modal analysis & Flexure & $170-3000$ & $22-43$ & Macroscopic & $10^{-4}$ \\
\hline HFV & Shear & $200-2000$ & $20-80$ & Macroscopic & $10^{-3}$ \\
\hline Nanoindentation & Indentation & $0.1-200$ & 20 & Nanoscopic & 5 \\
\hline SMM & Hertzian contact & $10^{4}-10^{5}$ & 20 & Microscopic & 4 \\
\hline Ultrasound & - & 10 & 20 & Macroscopic & - \\
\hline
\end{tabular}

The orange circles correspond to the modal analysis measurements. They show a correct general trend of the storage modulus in comparison to the DMA master curve and associated 2S2P1D model. In addition, modal tests allow the measurement of the reduced high-frequency loss factor, whereas the 2S2P1D model provides only a hypothetical asymptote. The identified loss factors are larger than those predicted by the model. Modal analysis is a 
pertinent experimental method to correctly estimate low-lossfactor values. The modal test implementation is simple, fast, inexpensive and non-destructive. It also offers the possibility to study structures with 'non-standard' geometries. This method has two main disadvantages. Firstly, only low temperature measurements can be performed because of the softening of the material above the glass transition. Secondly, a correlation is necessary between the experiment and the model, and the finite element model may be difficult to establish because of the uncertainties related to the experimental conditions. The purple dots correspond to the high frequency measurements using viscoanalysis. Despite the uncertainties detailed previously, Figure 9 shows that good agreement is found between the HFV results and the 2S2P1D model. As mentioned previously, there are unexpected trends in reference to $E^{*}$ and $\tan (\delta)$ at reduced frequencies because of the increased sample stiffness with values within the same order of magnitude as the jaw stiffness for this range of frequencies. There is also a slight frequency shift on the glass transition which can be observed on the loss factor plot. This can be explained by the temperature control, which is performed using a thermocouple sensor placed in the tBA/PEGDMA sample near the test bench. However, owing to thermal inertia of the bench, the precision of applied temperatures may be not optimal. Nevertheless, the general trend remains correct and this device is, therefore, capable-unlike other methods-of measuring values at the glass transition. Moreover, the HFV tests succeeded in measuring the impressive loss factor that was larger than two during the glass transition phase. Therefore, HFV is promising, and the tests on the tBA/PEGDMA have highlighted the experimental and analytical limits of the device.

The blue diamonds, squares, and dots, represent the measurements corresponding to nano-indentation, SMM, and ultrasonic tests, respectively. The results confirm the existence of the asymptote at reduced frequencies in the case of the 2S2P1D model. Hence, results validate the stabilisation of the storage modulus. The differences in the asymptotic values between the various methods, given the different scales of the tests, the multiple means of excitation, and the different levels of deformation, are minimal. Therefore, these experimental methods constitute a 
robust solution for determining the storage modulus in the glassy state. In addition, ultrasound tests as well as SMM analyses make it possible to estimate a Poisson's ratio value. These methods, without additional means of measurement, allow quick estimation of the Poisson's ratio close to the estimate obtained by DIC in the quasi-static regime. Conversely, none of these three methods allow the extraction of the loss factor at these reduced frequency levels. The dynamic nano-indentation method could access this, but the loss factor is too small and the phase shift between the excitation and response cannot be detected. The SMM method could collect information on the phase shift, but the exploitation of these results is not yet effective.

Finally, in spite of the different types of the loading modes employed herein, the different strain levels, and the different solicited scales (Table 2), all the experimental methods yielded consistent results. Various experimental methods have been used, from commercial to in-house and high-tech methods, yet the 2S2P1D model was consistent with all the seven tested experimental methods. For the materials that are thermorheologically simple, and for which the TTS principle is relevant, DMA can provide a reliable mechanical characterisation within a large band of frequencies and temperatures. However, for practical applications, where only one experimental test is possible, it seems reasonable to choose the experimental method that is adjusted to the targeted structural application in order to limit possible biases in the visco-elastic property estimation.

\section{Conclusions}

Broadband characterisation of the shape memory polymer tBA/PEGDMA was performed using seven experimental methods. Dynamic mechanical analyses allowed the determination of the master curve and a suitable visco-elastic model based on the timetemperature superposition. The quasi-static tests in the glassy state validated the asymptote on the storage modulus at reduced frequencies. The modal analyses at various temperatures provided values that were close to the glass transition and estimated nonzero values for the loss factor at reduced frequencies. The tests on the HFV bench characterised the tBA/PEGDMA polymer within a 
large band of temperatures, and highlighted the fact that the loss factor achieved values that were larger than two at the glass transition phase. The complementary methods, nano-indentation, scanning micro-deformation microscopy and ultrasound, also validated the stabilisation of the storage modulus in the glassy state. Finally, this work provided a wide frequency and temperature mechanical characterisation of the visco-elastic mechanical properties of the tBA/PEGDMA thanks to the various experimental methods employed herein at different scales.

\section{Acknowledgment}

The work of Pauline Butaud was co-financed by The French National Research Agency under Grant No. ANR-12-JS09-008COVIA. It has been performed in cooperation with the Labex ACTION program (ANR-11-LABX-0001-01).

\section{Data availability}

The raw/processed data required to reproduce these findings cannot be shared at this time due to technical limitations.

\section{References}

[1] P. Grootenhuis, The control of vibrations with viscoelastic materials, Journal of Sound and Vibration 11 (4) (1970) 421-433.

[2] A. Arajo, C. M. Soares, C. M. Soares, J. Herskovits, Optimal design and parameter estimation of frequency dependent viscoelastic laminated sandwich composite plates, Composite Structures 92 (9) (2010) 2321 - 2327, fifteenth International Conference on Composite Structures. 
[3] P. Aumjaud, C. Smith, K. Evans, A novel viscoelastic damping treatment for honeycomb sandwich structures, Composite Structures 119 (2015) 322 - 332.

[4] P. Butaud, E. Foltête, M. Ouisse, Sandwich structures with tunable damping properties: on the use of shape memory polymer as viscoelastic core, Composite Structures 153 (2016) 401-408.

[5] J. Diani, P. Gilormini, G. Agbobada, Experimental study and numerical simulation of the vertical bounce of a polymer ball over a wide temperature range, Journal of Materials Science 49 (5) (2014) 2154-2163.

[6] W. Stark, M. Jaunich, J. McHugh, Dynamic mechanical analysis (dma) of epoxy carbon-fibre prepregs partially cured in a discontinued autoclave analogue process, Polymer Testing 41 (2015) 140-148.

[7] J. Hu, W. Chen, P. Fan, J. Gao, G. Fang, Z. Cao, F. Peng, Epoxy shape memory polymer (smp): Material preparation, uniaxial tensile tests and dynamic mechanical analysis, Polymer Testing 62 (2017) 335 - 341.

[8] M. L. Williams, R. F. Landel, J. D. Ferry, The temperature dependence of relaxation mechanisms in amorphous polymers and other glass-forming liquids, Journal of the American Chemical Society 77 (14) (1955) 3701- 3707.

[9] J. Seitz, C. Balazs, Application of time-temperature superposition principle to long term engineering properties of plastic materials, Polymer Engineering \& Science 8 (2) (1968) 151-160.

[10] S. Hutcheson, G. McKenna, in: 6th International Conference on Mechanics of Time Dependent Materials Conference, Monterey, California, 2008. 
[11] J. Diani, P. Gilormini, On necessary precautions when measuring solid polymer linear viscoelasticity with dynamic analysis in torsion, Polymer Testing 63 (2017) 275-280.

[12] P. Butaud, V. Placet, J. Klesa, M. Ouisse, E. Foltête, X. Gabrion, Investigations on the frequency and temperature effects on mechanical properties of a shape memory polymer (veriflex), Mechanics of Materials 87 (2015) 50-60.

[13] Y. Tsai, C.-h. Tai, S.-J. Tsai, F.-J. Tsai, Shape memory effects of poly (ethylene terephthalate-co-ethylene succinate) random copolymers, European Polymer Journal 44 (2) (2008) 550-554.

[14] M. J. Barwood, C. Breen, F. Clegg, C. L. Hammond, The effect of organoclay addition on the properties of an acrylate based, thermally activated shape memory polymer, Applied Clay Science 102 (2014) 41-50.

[15] D. P. Nair, N. B. Cramer, T. F. Scott, C. N. Bowman, R. Shandas, Photopolymerized thiol-ene systems as shape memory polymers, Polymer 51 (19) (2010) 4383-4389.

[16] C. M. Yakacki, R. Shandas, C. Lanning, B. Rech, A. Eckstein, K. Gall, Unconstrained recovery characterization of shape-memory polymer networks for cardiovascular applications, Biomaterials 28 (14) (2007) 2255 - 2263.

[17] E. Wornyo, K. Gall, F. Yang, W. King, Nanoindentation of shape memory polymer networks, Polymer 48 (11) (2007) 3213-3225.

[18] A. M. Ortega, S. E. Kasprzak, C. M. Yakacki, J. Diani, A. R. Greenberg, K. Gall, Structure-property relationships in photopolymerizable polymer networks: Effect of composition on the crosslinked structure and resulting thermomechanical 
properties of a (meth) acrylate-based system, Journal of applied polymer science 110 (3) (2008) 1559-1572.

[19] S. E. Zeltmann, K. A. Prakash, M. Doddamani, N. Gupta, Prediction of modulus at various strain rates from dynamic mechanical analysis data for polymer matrix composites, Composites Part B: Engineering 120 (2017) 2734 .

[20] V. Srivastava, S. a. Chester, L. Anand, Thermally actuated shape-memory polymers: Experiments, theory, and numerical simulations, Journal of the Mechanics and Physics of Solids 58 (8) (2010) 1100-1124.

[21] P. Butaud, M. Ouisse, V. Placet, E. Foltête, Experimental investigations on viscoelastic properties of a shape memory polymer, in: ASME SMASIS2014, American Society of Mechanical Engineers, 2014.

[22] ASTM D638-03, Standard test method for tensile properties of plastics.

[23] J. Réthoré, F. Hild, S. Roux, Shear-band capturing using a multiscale extended digital image correlation technique, Computer Methods in Applied Mechanics and Engineering 196 (49) (2007) 5016-5030.

[24] J. Mottershead, M. Friswell, Model updating in structural dynamics: a survey, Journal of sound and vibration 167 (2) (1993) 347-375.

[25] F. Renaud, G. Chevallier, J. L. Dion, R. Lemaire, Viscoelasticity measurement and identification of viscoelastic parametric models, in: ASME International Design Engineering Technical Conferences and Computers and Information in Engineering Conference, Washington DC, 2011, pp. 701-708. 
[26] F. Renaud, Etude de l'incidence des comportements dissipatifs dans les instabilités vibratoires des systèmes de freinages, Ph.D. thesis, Châtenay Malabry, Ecole centrale de Paris (2011).

[27] W. C. Oliver, G. M. Pharr, An improved technique for determining hardness and elastic modulus using load and displacement sensing indentation experiments, Journal of materials research 7 (06) (1992) 1564-1583.

[28] X. Li, B. Bhushan, A review of nanoindentation continuous stiffness measurement technique and its applications, Materials characterization 48 (1) (2002) 11-36.

[29] P. Vairac, B. Cretin, Scanning microdeformation microscopy in reflection mode, Applied physics letters 68 (4) (1996) 461-463.

[30] B. Cretin, F. Sthal, Scanning microdeformation microscopy, Applied physics letters 62 (8) (1993) 829-831.

[31] J. Le Rouzic, P. Delobelle, B. Cretin, P. Vairac, F. Amiot, Simultaneous measurement of young's modulus and poisson's ratio at microscale with two-modes scanning microdeformation microscopy, Materials Letters 68 (2012) 370-373.

[32] N. I. M. Yusoff, D. Mounier, G. Marc-Stephane, M. R. Hainin, G. D. Airey, H. D. Benedetto, Modelling the rheologicalproperties of bituminous binders using the $2 \mathrm{~s} 2 \mathrm{p} 1 \mathrm{~d}$ model, Construction and Building Materials 38 (0) (2013) 395 $-406$.

[33] R. Caracciolo, M. Giovagnoni, Frequency dependence of poisson's ratio using the method of reduced variables, Mechanics of materials 24 (1) (1996) 75-85. 
[34] S. Mousavi, D. Nicolas, B. Lundberg, Identification of complex moduli and poisson's ratio from measured strains on an impacted bar, Journal of Sound and Vibration 277 (4) (2004) 971-986.

[35] T. Pritz, The poisson's loss factor of solid viscoelastic materials, Journal of Sound and Vibration 306 (3) (2007) 790802.

[36] G. N. Greaves, A. Greer, R. Lakes, T. Rouxel, Poisson's ratio and modern materials, Nature materials 10 (11) (2011) 823-837.

[37] D. J. O'Brien, N. R. Sottos, S. R. White, Cure-dependent viscoelastic poisson's ratio of epoxy, Experimental Mechanics 47 (2) (2007) 237-249.

[38] H. Oberst, K. Frankenfeld, Uber die da"mpfung der biegeschwingungen" du"nner bleche durch fest haftende bel"age, Acta Acustica united with Acustica 2 (Supplement 2) (1952) 181-194.

[39] ASTM 756-9 : Standard test method for measuring vibration-damping properties of materials (1998).

[40] H. Festjens, C. Gaël, R. Franck, D. Jean-Luc, L. Remy, Effectiveness of multilayer viscoelastic insulators to prevent occurrences of brake squeal: A numerical study, Applied Acoustics 73 (11) (2012) 1121-1128. 


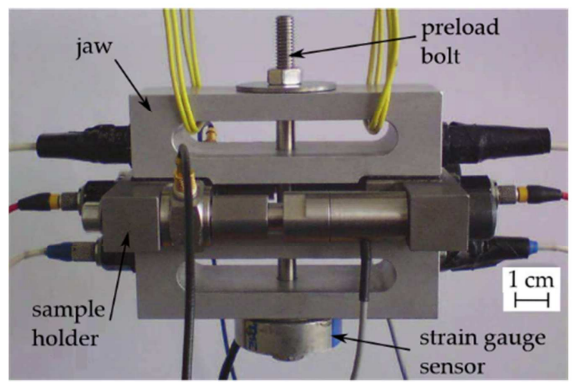

(a) HFV system [26]

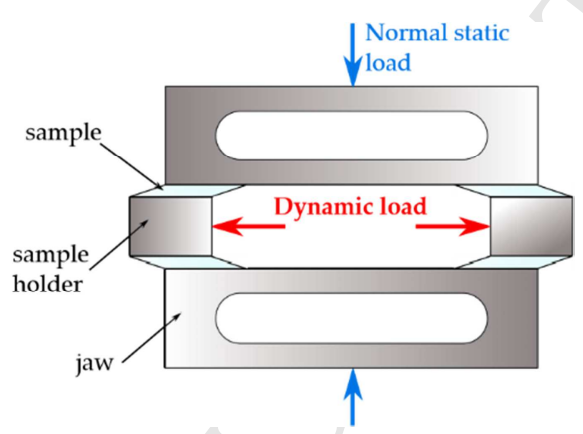

(b) HFV schematic principle

Figure 1: High-frequency cisco-analyser.

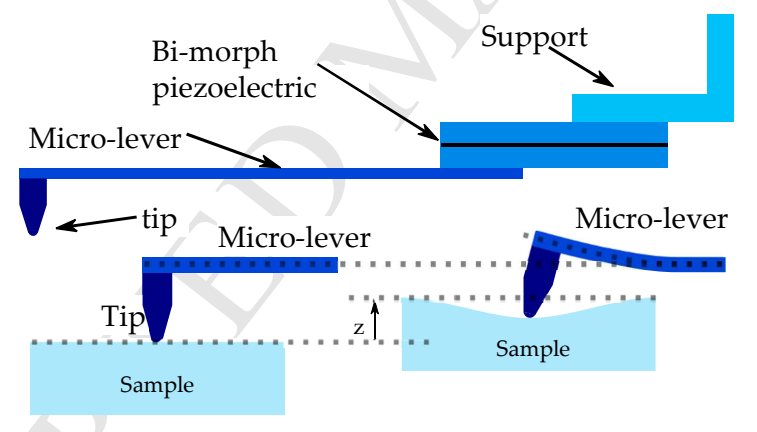

Figure 2: SMM principle showing the tip in contact with the sample. 


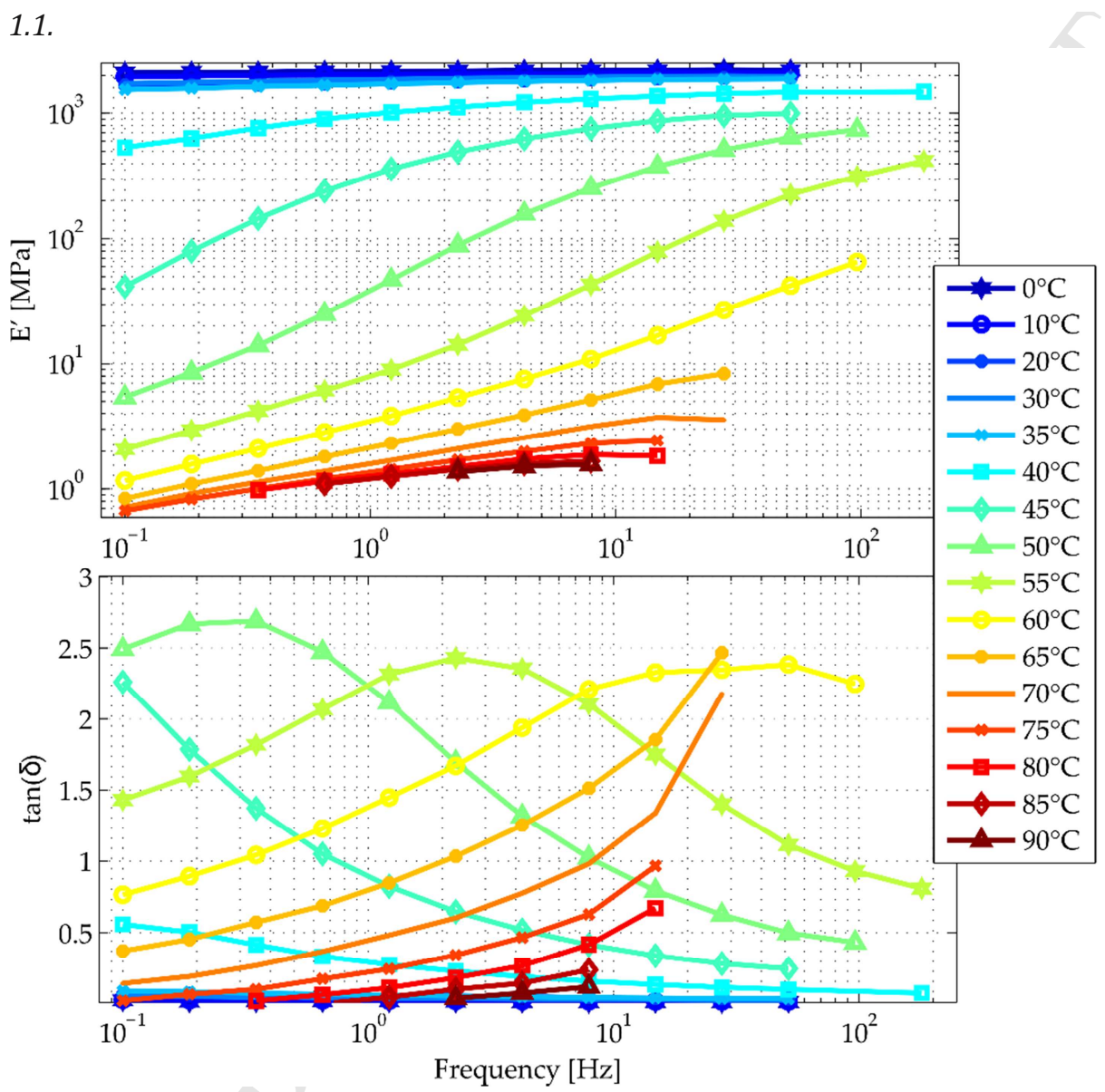

Figure 3: Plots of the variations in the storage modulus $E^{\prime}$ and loss factor $\tan (\delta)$ of the tBA/PEGDMA polymer as a function of frequency and for different temperatures measured using dynamic mechanical analysis. 


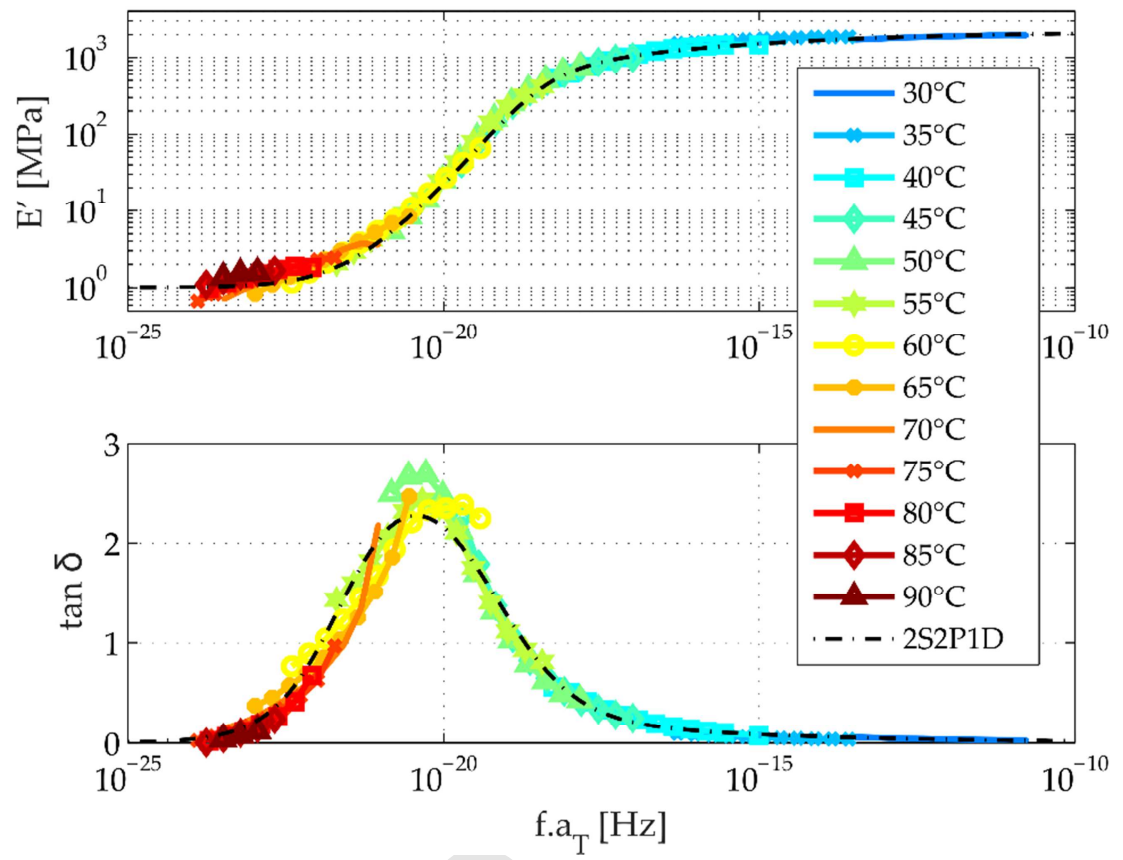

Figure 4: Master curve from DMA measurements and 2S2P1D model for the storage modulus and the loss factor of the tBA/PEGDMA, according to the reduced frequency $f . a_{T}$ with $T_{0}=$ $20^{\circ} \mathrm{C}$. 

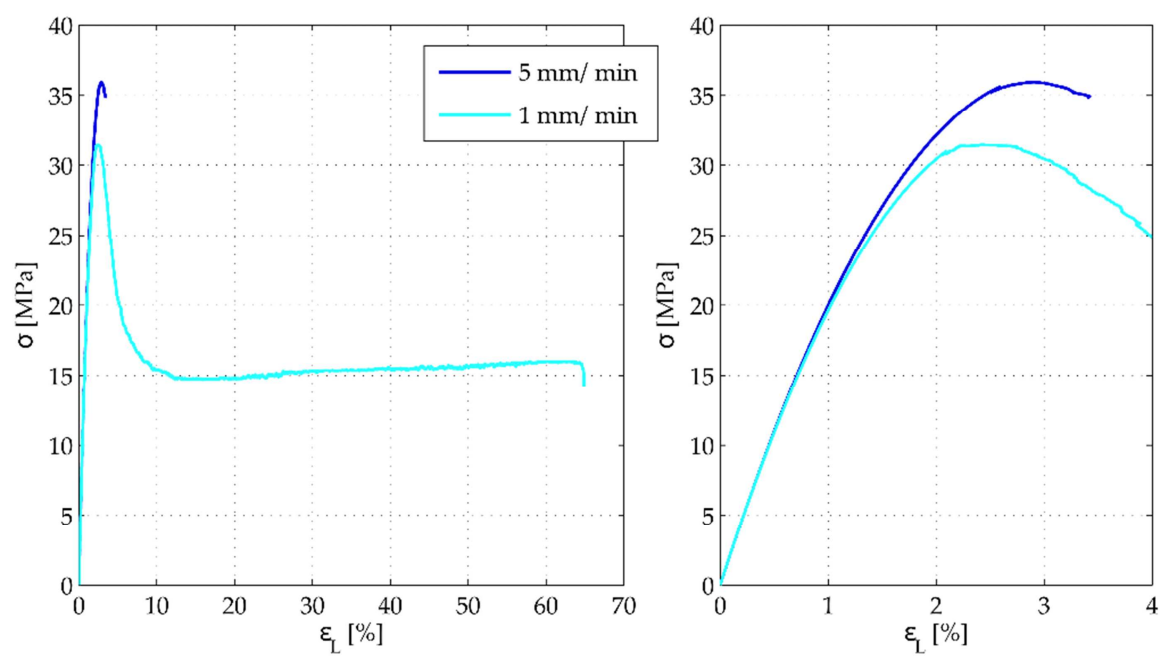

Figure 5: Nominal stress according to the engineering strain for two loading rates. (a) Evidence of ductile or brittle fragile behaviour according to the loading rate. (b) Enlarged view of nominal stress and strain for longitudinal strain in the range of 0 and $4 \%$.
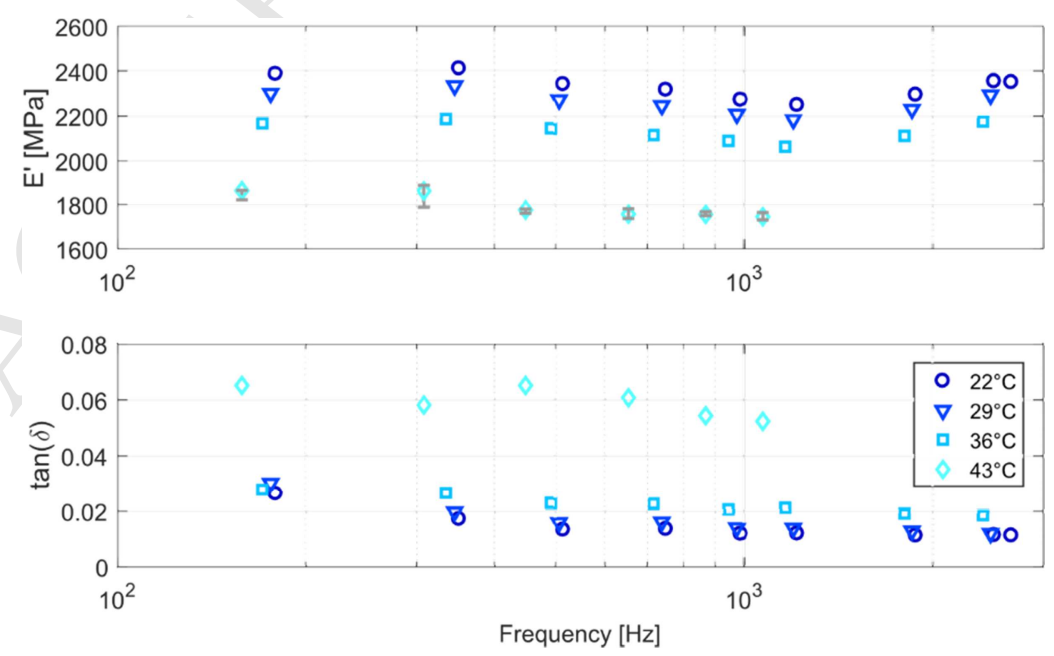
Figure 6: Storage modulus and loss factor estimated based on modal analyses for different temperatures, as a function of frequency.

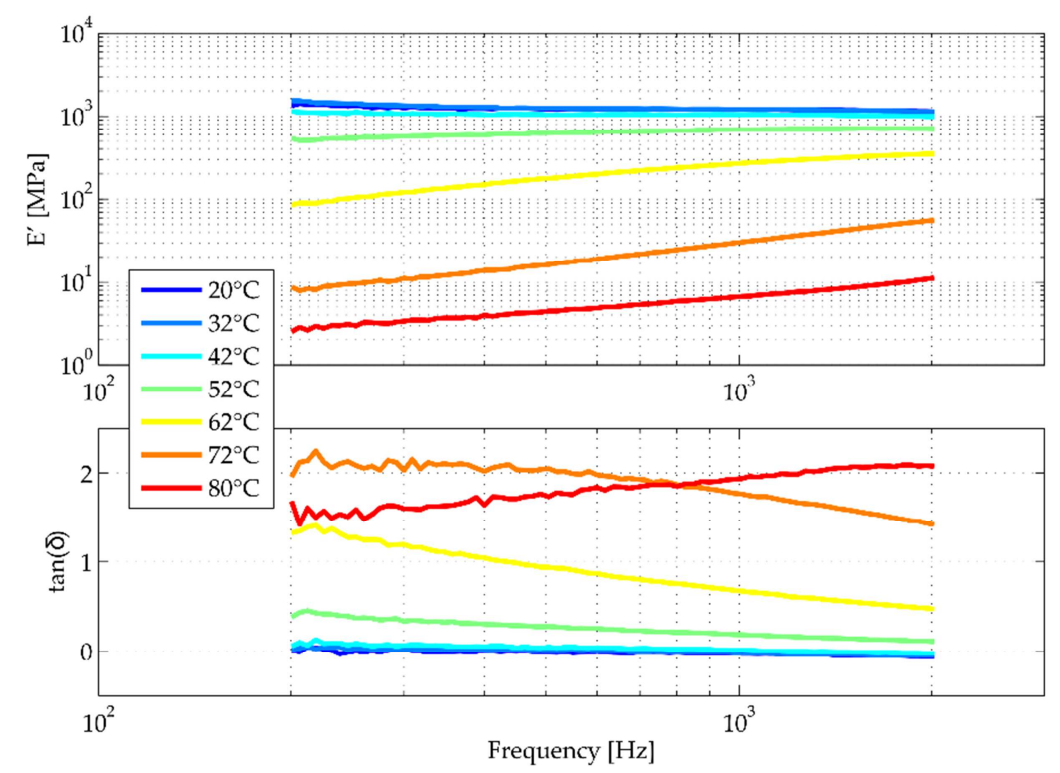

Figure 7: Storage modulus and loss factor measured by HFV for different temperatures as a function of frequency.

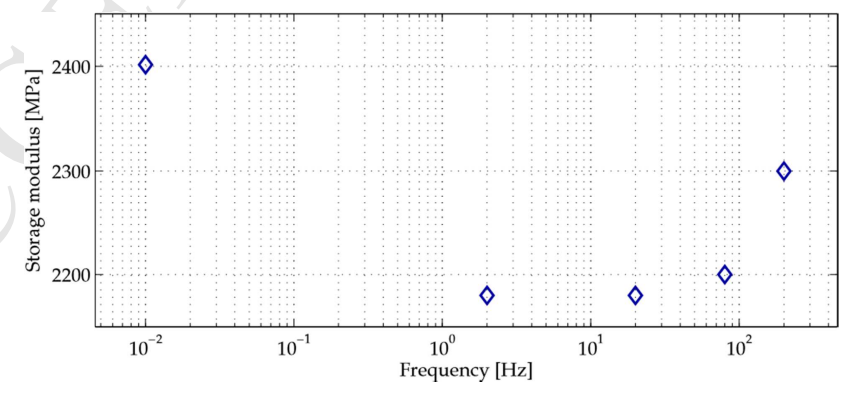

Figure 8: Storage modulus as a function of the frequency measured by nano-indentation at $20^{\circ} \mathrm{C}$. 


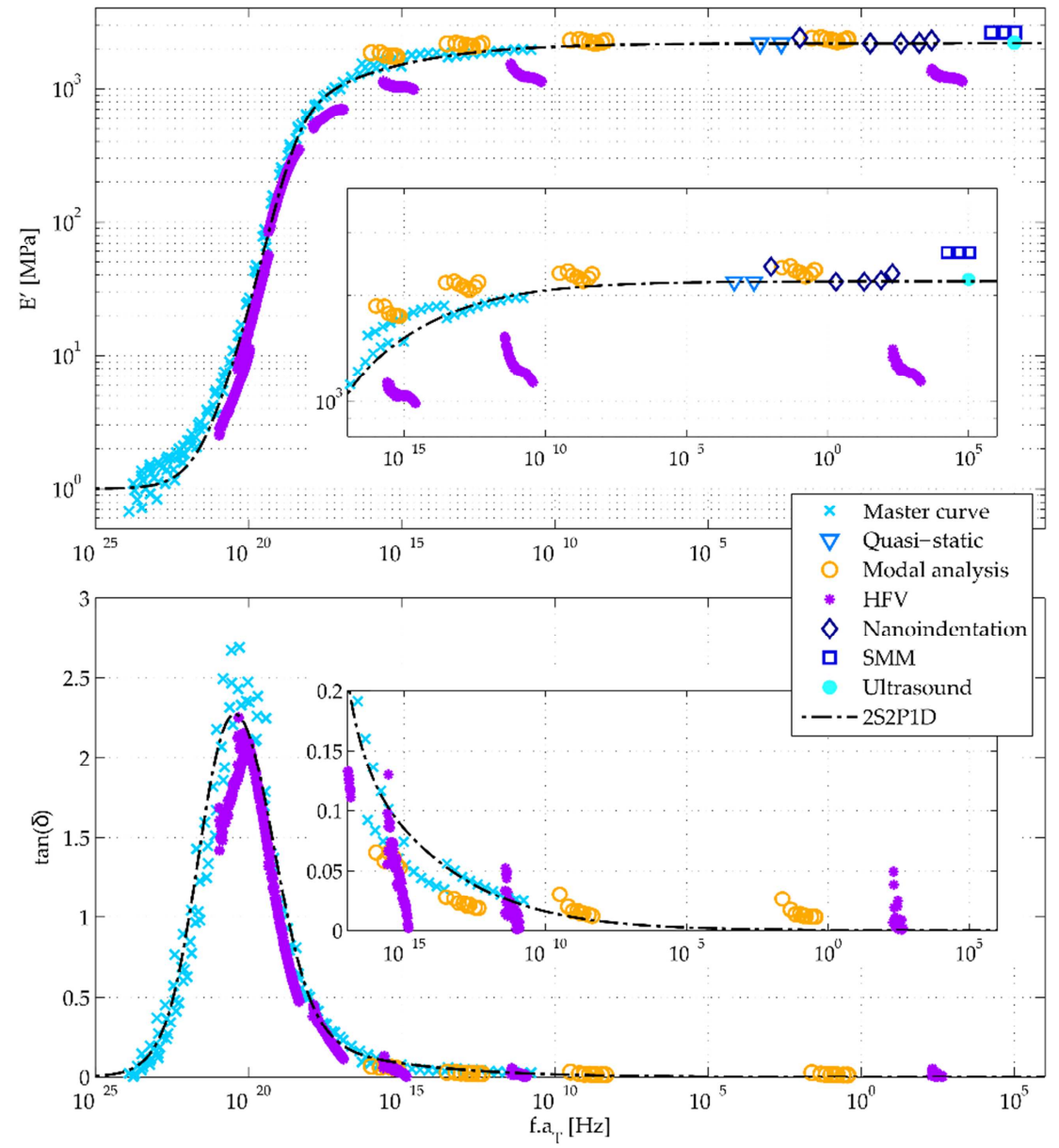

Figure 9: Dynamic characterisation benchmark of the tBA/PEGDMA. Variations in the storage modulus and loss factor according to the reduced frequency at $20^{\circ} \mathrm{C}$, as measured by the seven experimental methods employed herein, and as represented by the 2S2P1D visco-elastic model. 
we perform a wideband characterization of the tBA/PEGDMA Shape Memory Polymer ;

we identified a viscoelastic model derived from the dynamic mechanical analysis ;

we compare various identification methods of the mechanical properties of the tBA/PEGDMA ;

we compare the predicted viscoelastic properties through seven experimental methods ;

we validate the continuity of viscoelastic properties of a polymer identified from multi loading modes on a wide frequency-temperature-scale range 